\title{
Patterns of source monitoring bias in incarcerated youths with and without conduct
} problems

Larisa Morosan ${ }^{1,2}$, Deborah Badoud ${ }^{1,2}$, George Salaminios $^{3}$, Stephan Eliez ${ }^{2}$, Martial Van der Linden ${ }^{4}$, Patrick Heller ${ }^{5}$, Martin Debbané1, 2,4

\footnotetext{
${ }^{1}$ Developmental Clinical Psychology Unit, Faculty of Psychology, University of Geneva, Switzerland

${ }^{2}$ Developmental Imaging and Psychopathology Lab, Office Médico-Pédagogique, Department of Psychiatry, University of Geneva, Switzerland

${ }^{3}$ Research Department of Clinical, Educational and Health Psychology, University College London, UK

${ }^{4}$ Psychopathology and Cognitive Neuropsychology Unit, University of Geneva, Switzerland

${ }^{5}$ Department of Forensic Medicine and Psychiatry, Department of communitarian, emergency and first aid medicine and psychiatry, Department of mental health and psychiatry, University Hospital of Geneva
}

\section{Corresponding Author:}

Larisa Morosan

University of Geneva,

40, Bd du Pont-d'Arve,

1211 Genève

Email: Larisa.Morosan@unige.ch

Tel.: + $41(0) 223790606$

\section{Authors' e-mail adresse :}

Deborah Badoud:

Deborah.Badoud@unige.ch

George Salaminios:

g.salaminios@ucl.ac.uk

Stephan Eliez: stephan.eliez@etat.ge.ch

Martial Van der Linden:

Martial.VanDerLinden@unige.ch

Patrick Heller: Patrick.Heller@hcuge.ch

Martin Debbané:

Martin.Debbane@unige.ch

Keywords : adolescence, antisocial, self monitoring, self-awareness, delinquency 


\begin{abstract}
Introduction: Antisocial individuals present behaviors that violate the social norms
\end{abstract} and the rights of others. In the present study, we examine whether biases in monitoring the self-generated cognitive material might be linked to antisocial manifestations during adolescence. We further examine the association with psychopathic traits and conduct problems (CP).

Methods: Sixty-five incarcerated adolescents (IA; Mage=15.85, $S D=1.30)$ and 88 community adolescents $(\mathrm{CA} ; M$ age $=15.78, S D=1.60)$ participated in our study. In the IA group, 28 adolescents presented CP $($ Mage $=16.06, S D=1.41)$ and 19 did not meet the diagnostic criteria for $\mathrm{CP}(M a g e=15.97, S D=1.20)$. Source monitoring was assessed through a speech-monitoring task, using items requiring different levels of cognitive effort; recognition and source-monitoring bias scores (internalizing and externalizing biases) were calculated.

Results: Between group comparisons indicate greater overall biases and different patterns of biases in the source monitoring. IA participants manifest a greater externalizing bias, whereas CA participants present a greater internalizing bias. In addition, IA with CP present different patterns of item recognition.

Conclusions: These results indicate that the two groups of adolescents present different types of source-monitoring bias for self-generated speech. Future studies may examine the developmental implications of self-monitoring biases in the perseverance of antisocial behaviors from adolescence to adulthood. 


\section{Introduction}

2 Antisocial behavior entails a range of violations to the moral and physical integrity or the

3 property of others, and more broadly to social norms. These manifestations lead to a variety

4 of research topics targeting phenomena such as aggression, behavioral disorders, and

5 delinquency. In the field of child and adolescent psychiatry, diagnoses such as conduct

6 disorder or oppositional defiant disorder are employed to describe different types of antisocial

7 behaviors (Moffitt, Caspi, Harrington, \& Milne, 2002). Several authors group these

8 psychiatric diagnoses under the concept of conduct problems (CP) (Hill, 2002; Schwenck et

9 al., 2014), which are more prevalent among incarcerated youth (Köhler, Heinzen, Hinrichs, \&

10 Huchzermeier, 2009), and appear to be associated with the development of life-course

11 persistent antisocial behavior (Moffitt, 1993; Sevecke, Kosson, \& Krischer, 2009). In

12 addition, personality researchers have demonstrated that psychopathy, defined as the lack of

13 affectivity, deceitful interpersonal style and impulsive and irresponsible behavior, may sustain

14 antisocial manifestations (Andershed, Kerr, Stattin, \& Levander, 2002). The developmental

15 trajectories of antisocial individuals are marked by serious personal, social, and educational

16 difficulties, and the damage resulting from their behaviors result in important costs for the

17 society (Morgado \& Vale-Dias, 2013).

18 For this purpose, a large body of research focuses on the psychological processes that might

19 underlie antisocial manifestations. Some authors propose that antisocial individuals present

20 impairments in the monitoring of their own actions (Bernat, Nelson, Steele, Gehring, \&

21 Patrick, 2011; Brazil et al., 2009; Hall, Bernat, \& Patrick, 2007; Vilà-Balló, Hdez-Lafuente,

22 Rostan, Cunillera, \& Rodriguez-Fornells, 2014). For example, a series of studies using

23 electroencephalography methodologies indicate that antisocial individuals present lower

24 activation of the error related negativity, an indicator of action monitoring and error detection

25 processes (Bernat et al., 2011; Brazil et al., 2009; Hall et al., 2007; Vilà-Balló et al., 2014). 
These results suggest impairments in matching the expected outcome of their own actions to the actual outcome, leading to impairments in monitoring their own behaviors (Vilà-Balló et al., 2014).

A key cognitive process involved in the monitoring of one's behaviors is the ability to discriminate between different sources of information, traditionally studied within the sourcemonitoring framework (Johnson, Hashtroudi, \& Lindsay, 1993). Different types of source monitoring processes have previously been described: internal-external source monitoring, which enables one to distinguish between information generated by oneself from information generated by another person; external source monitoring, which refers to the ability to distinguish between two external sources; and internal source monitoring -distinguishing between what one imagined doing or saying from what one actually did or said (Johnson et al., 1993). Biases in the self-monitoring can arise as a result of several factors. The sourcemonitoring framework postulates that the amount and the clarity of sensorial signals (sensorial precision) biases towards an external attribution of the source. On the other hand, the amount and clarity of cognitive signals, such as thoughts, internal speech, imagination (cognitive precision) biases towards an internal attribution of the source of the material (Johnson et al., 1993).

43 The source monitoring framework can be informed by the forward model of motor control proposed by Miall and Wolpert, (1996). This model was initially developed to conceptualize the monitoring of actions, however, recent studies adapted it for the monitoring of thought content, such as internal speech (Frith, Blakemore, \& Wolpert, 2000; Jones \& Fernyhough, 2006). The forward model postulates that the correct attribution of the source results from a match between the predicted and the actual sensorial consequences of the action. On the other hand, a mismatch leads to biases in the attribution of the source. This mismatch might result due to interferences at different levels: in generating the prediction of the sensorial outcome 
of the action or in the processing of the actual sensorial feedback of the action (Blakemore,

52 Oakley, \& Frith, 2003). Based on these two approaches, the source monitoring framework and the forward model, we can hypothesize that the impairments in the monitoring of behaviors presented by antisocial individuals might be explained by impairments in the source attribution. Considering the studies presented above describing impairments in processing the outcome of the action, we can hypothesize that the impairments in the source monitoring in antisocial individuals might be due to impairment in processing the sensorial feedback of their actions. This hypothesis comes in the continuity of several studies indicating impairments in sensorial integration in antisocial individuals (Assadi et al., 2007; Faruk, Demirel, Tayyib, \& Emül, 2016; Lindberg, Tani, Stenberg, \& Appelberg, 2004; Wang et al., 2016).

To the best of our knowledge, no study investigated the source monitoring of thought content in antisocial individuals. Thus, the present study focuses on self-generated speech monitoring, which represents a key component of internal source monitoring. We seek to explore the potential associations between the monitoring of self-generated speech and two crucial characteristics of antisocial individuals, $\mathrm{CP}$ and psychopathic traits. We focus on adolescence as a critical period for the development of antisocial tendencies (Frick \& White, 2008). In addition, the investigation of source monitoring in a group of incarcerated adolescents may help identify early factors sustaining these maladaptive behaviors, and could further inform early prevention and intervention strategies.

71 For this purpose, we employ a task that examines the participant's capacity to discriminate

72 between one's silently- and overtly produced speech. Previous studies indicate that the 73 cognitive effort of the stimuli might play an important role in the monitoring of the source of 74 the material (Debbané, Van der Linden, Glaser, \& Eliez, 2010; Larøi, Van Der Linden, \& 75 Marczewski, 2004; Sugimori \& Tanno, 2010). Thus, we manipulated the cognitive effort by 
presenting different types of stimuli, words and non-words (Debbané et al., 2010). This task differentiates between two types of monitoring biases; the externalizing bias, which consists in reporting silently generated speech as overtly produced; and the internalizing bias, which consists in reporting overtly generated speech as silently produced. Based on previous studies investigating the monitoring of behaviors in antisocial individuals and on the postulates of the source-monitoring framework, we hypothesize that the incarcerated group will present a greater biases in the self-generated speech monitoring and that the bias will be greater for the items that require greater cognitive effort, the non-words. Furthermore, we aim to explore the relationship between source-monitoring of self-generated speech and psychopathic traits in both groups. In addition, we propose to investigate the differences in source monitoring, between the incarcerated adolescents with and without CP.

\section{Method}

\section{Participants}

Sixty-five adolescents incarcerated (IA) in an observation and detention center for youths in Geneva, Switzerland, took part in the study (Mage $=15.85, S D=1.30 ; 20$ females). Eighty-eight community adolescents (CA) with no previous criminal convictions formed the comparison group (Mage=15.78, $S D=1.60 ; 30$ females). The $\mathrm{CA}$ were recruited via advertising leaflets and by word of mouth and were tested at our research unit. The IA were individually tested at the center facility in a private room.

The inclusion criteria were age (12-18 years) and fluency in French. In addition, the subjects with a history of psychotic disorders and intellectual deficiency were not included in the study. For administrative reasons, information about the reason for incarceration was available for 60 of the IA; the majority committed more than one criminal offense, including physical and verbal aggression (16.7\%), drug-related crimes (35\%), theft and robbery $(45 \%)$, runaways and risky behaviors $(33.3 \%)$, conduct difficulties $(20 \%)$ and driving violations 
101 (8.3\%). In relation to availability of the detained adolescents, forty-seven IA could be screened for psychiatric problems according to DSM-IV criteria using the Kiddie-SADS Present and Lifetime Version (K-SADS-PL) semi-structured interview (Kaufman et al., 1997). Trained clinical psychologists from our team conducted the interview under the supervision of MD. Diagnostic information is reported in Table 1. On the basis of the clinical 106 interview, two IA subgroups were created: conduct problems (CP) group, which includes the twenty-eight IA who met the criteria for CD or ODD ( 7 females, Mage=15.97, $S D=1.20$ ), and non-CP group, which includes the nineteen IA who did not meet any of the conduct problems diagnostic criteria ( 7 females, Mage $=16.06, S D=1.41$ ).

All the participants completed the full protocol, except for one participant from the IA group

111 who didn't complete the Youth Self-Report. Written informed consent was obtained from all

112 the participants and, for participants under 18 years old, also from their legal guardians. The 113 protocol was approved by the Institutional Review Board of the Department of Psychiatry of 114 the University of Geneva Medical School. The adolescents in both groups received monetary 115 compensation for their participation in the study.

\section{Instruments}

117 Source-monitoring task

118 Source-monitoring was investigated using a self-generated speech-monitoring task, the 119 word/non-word task (Debbané et al., 2010). The task consisted of two parts: a reading 120 procedure, followed by an incidental recognition and source monitoring procedure. In the first 121 part of the task was presented as a reading and pronunciation exercise. The participants were 122 required to read, either aloud or silently, a series of words (low cognitive effort items) or non123 words (high cognitive effort items) presented on a computer screen. They were instructed to 124 pay special attention to their pronunciation, even when reading the items silently; they were 125 not informed that a recognition and source monitoring procedure would follow. After two 
exercise trials, making sure that the subjects understood the task, six blocks (six silent, six

127 aloud) of eight items (eight words, eight non-words) were randomly presented. In total, each

128 condition contained 12 items, for a total of 48 items (12 words, 12 non-words read aloud and

12912 words, 12 non-words silently read). After a 10-15 minutes visuospatial filler task, the

130 second part of the task was introduced. A recognition sheet was then handed out, containing

13172 items (the 48 items read in the first part of the task, plus 12 new word and 12 new non-

132 word items). The participants were instructed that they have to indicate which items from the

133 recognition list had appeared in the reading phase (yes/no- recognition test), and to attribute

134 them to a reading condition (read silently or aloud- monitoring test). By using two types of

135 items, the task aimed to differentiate the monitoring of self-generated speech in two different

136 cognitive effort levels, high cognitive effort (non-words) and low cognitive load (words).

137 For the recognition phase, signal detection theory (Stanislaw \& Todorov, 1999) was used to

138 assess the sensibility for each type of items (word and non-words) for both reading conditions

139 (aloud and silently). The estimation of d-prime scores were calculated by subtracting the $z$

140 score corresponding to the false alarms from the $z$ score corresponding to hit rate. False

141 recognition scores were calculated as the number of words that were not presented in the

142 reading phase of the task (distractors), misrecognized as belonging to the reading phase.

143 Higher d-prime scores indicate a better recognition accuracy.

144 In order to assess the monitoring bias, externalizing and internalizing bias scores were

145 calculated. The externalizing bias was calculated by dividing the total score for items read

146 silently, but identified as read aloud in the monitoring test, out of the total score of items

147 correctly recognized as read silently. In the same way, the internalizing bias score was

148 calculated by dividing the total score of items read overtly but identified as read silently out

149 of the total score of items correctly recognized as read overtly. Externalizing and internalizing 
150 bias scores were calculated overall, as well as for each item type (word and non-word)

151 separately.

152 Self-report questionnaires

153 Externalizing (including aggressive behaviors and rule-breaking behaviors) and internalizing 154 (including withdrawal, anxiety, depression, and somatic complains) problems in participants 155 aged $<18$ years were assessed using the Youth Self-Report (YSR; Achenbach,

156 1991).(Achenbach, 1991) For the participants aged $\geq 18$ but $<19$ years, the Adult Self-Report 157 (ASR; Achenbach and Rescorla, 2003)(Achenbach \& Rescorla, 2003) was used. Each of the 158119 items in these instruments is evaluated on a 3-point scale, with 0 corresponding to "not true", 1 to "sometimes true" and 2 to "very or often true".

160 Psychopathic traits were assessed using the French version of the Youth Psychopathic 161 Inventory (YPI; Andershed et al., 2002). The YPI evaluates three dimensions of psychopathy, 162 each consisting of several subscales: an interpersonal dimension assessing grandiose, 163 manipulative behaviors, an affective dimension assessing callous-unemotional traits, and a 164 dimension assessing impulsive, irresponsible behavior. The 50 items of the YPI are scored on 4-point scale, from 1 corresponding to "does not apply at all” to 4 corresponding to "applies 166 very well”.

167 In order to assess the cognitive functioning, we used the French versions of two subtests,

168 Vocabulary and Digit Span, of the Wechsler Intelligence Scale for Children-Fourth edition 169 (WISC; Wechsler, 2003) (Wechsler, 2003) and, for participants $\geq 18$ years old, the Wechsler 170 Adult Intelligence Scale-Third edition (WAIS; Wechsler, 1997).(Wechsler, 1997) The

171 Vocabulary subtest measures word knowledge, language development, and concept

172 understanding, whereas the Digit Span subtest investigates the short-term memory 173 performances. 


\section{Statistical analysis}

$175 T$-test analyses were conducted for sample characteristics, such as the age, WISC/ WAIS

176 subscales, and YPI scores. Because the groups differ on WISC/WAIS subscales scores, and to

177 control for the potential effect of gender, both variables were entered as covariates in the

178 following analysis. For the self-monitoring task, mixed analysis of covariance (ANCOVA)

179 was conducted on the d-prime scores for each type of stimuli (words vs. non-words) on each

180 reading condition (aloud vs. silently), with group (IA vs. CA) as between factor. Mixed

181 ANCOVAs were conducted on the monitoring bias scores (externalizing vs. internalizing), for

182 each type of stimuli (word vs. non-word) and overall, with group (IA vs. CA) as between

183 factor. Partial correlation analysis was used to investigate the relationships between the

184 dependent variables and the sample characteristics, using gender as covariate.

185 In order to further explore the effects of the CP on the monitoring bias, we conducted the

186 same analyses to compare the subgroups of IA with without CP. Because the two subgroups

187 did not differ in the scores of the WISC/WAIS subscales, only gender was used as covariate.

188 Statistical analyses were carried out in SPSS, version 23 for Mac (SPSS Inc.,USA).

\section{Results}

\section{IA vs. CA groups}

\section{Sample characteristics}

192 Table 2 presents the results for the $t$-test analyses of the sample characteristics. In comparison

193 with the CA group, the IA group had significantly lower scores for the Vocabulary

$194(t(151)=5.71, p<0.001, d=0.92)$ and Digit $\operatorname{Span}(t(151)=2.21, p=0.020, d=0.36)$ subtests,

195 significantly higher scores for the externalizing subscale in the YSR/ASR $(t(150)=-8.10$,

$196 p<0.001, d=1.32$ ), and significantly higher scores for the impulsive, irresponsible behavior

197 subscale of the YPI $(t(146)=-6.14, p<0.001, d=1.01)$ and the callous-unemotional subscale 
$198(t(146)=-2.34, p=0.020, d=0.38)$. The groups did not differ in the mean age $(t(151)=-0.27$, $199 p=0.780, d=0.04)$, in the mean of the internalizing subscale of the YSR/ASR $(t(151)=0.90$, $200 p=0.360, d=0.14)$.

\section{Source-monitoring task results}

202 Results of mixed ANCOVA conducted on the d-prime scores, with group (IA vs. CA) as 203 between factor, and gender and both WISC/WAIS subscales scores as covariates, reveal a 204 main effect of reading condition $\left(F(1,148)=15.802, p<0.001\right.$, partial $\left.\eta^{2}=0.096\right)$ and a 205 significant effect of the item type $\left(F(1,148)=6.046, p=0.015\right.$, partial $\left.\eta^{2}=0.039\right)$ suggesting 206 that, independently of the group, silently read items and non-words are less accurately 207 recognized. The results indicate no interaction effect and no group effect $(p>0.05)$. Table 3 208 presents the means and the standard deviations for the d-prime scores, for each type of item in 209 both reading conditions.

210 The results of mixed ANCOVA conducted on the monitoring bias scores for each type of 211 item, with group (IA vs. CA) as between factor, revealed a main effect of item type ( $F(1$, $212148)=5.003, p=0.027$, partial $\left.\eta^{2}=0.033\right)$, indicating that the monitoring bias is greater for the 213 non-words. In addition, the results demonstrate a significant interaction effect between the

214 monitoring bias and the type of item $\left(F(1,148)=4.85, p=0.029\right.$, partial $\left.\eta^{2}=0.032\right)$, suggesting 215 that, independently of the group, the monitoring bias affects differently the type of items. To 216 follow up this interaction effect, simple effects were analyzed, revealing that, independently 217 of group, there was a greater internalization bias for non-words than for words $(F(1$, $218148)=12.628, p=0.001$, partial $\left.\eta^{2}=0.064\right)$, and greater internalization bias than externalization 219 bias for non-words $\left(F(1,148)=5.211, p=0.024\right.$, partial $\left.\eta^{2}=0.034\right)$. The results also indicate a main effect of the group $\left(F(1,148)=5.356, p=0.026\right.$, partial $\left.\eta^{2}=0.026\right)$, suggesting that the IA 221 group present more monitoring bias independently of the item type and bias. 
222 Finally, a significant triple interaction effect between the group, the monitoring bias, and the

223 item type $\left(F(1,148)=8.50, p=0.004\right.$, partial $\left.\eta^{2}=0.054\right)$ has been found, suggesting that the

224 interaction between the monitoring bias and the type of items was different in the two groups

225 of participants. Simple interaction effects were analyzed, indicating that, relative to the CA

226 group, the IA presented a significantly greater externalizing bias for non-words $(F(1$,

$227148)=10.120, p=0.002$, partial $\left.\eta^{2}=0.064\right)$, and significantly greater internalizing bias for the

228 words $\left(F(1,148)=5.088, p=0.026\right.$, partial $\left.\eta^{2}=0.033\right)$. In addition, the results suggested that the

229 CA presented greater internalizing bias for non-words than for words $(F(1,148)=16.017$,

$230 p<0.001$, partial $\left.\eta^{2}=0.098\right)$. These results are presented in the Figure 1.

\section{Correlation analysis}

232 We conducted partial correlations on the source monitoring results and YPI subscales, with

233 gender as covariate. For the d-prime scores in the recognition phase, no result exceeded the

234 significance level $(p>0.65)$. For the monitoring bias scores, in the CA group, the results did

235 not show any significant result $(p>0.196)$. In the IA group, the results reveal that the

236 internalizing bias for non-words was negatively correlated with interpersonal problems

237 subscale of the YPI $(r=-0.277, p=0.030)$. After the Bonferroni correction, no correlation

238 reached the significance level $(p=0.004)$.

239 CP vs. non-CP groups

240 Sample characteristics

241 The results of $t$-test analysis indicated that, relative to the non-CP group, the CP group

242 presented higher scores for the externalizing subscale of YSR/ASR $(t(44)=3.214, p=0.002$,

$243 d=0.96)$. The two groups did not differ in the mean age $(t(45)=-0.237, p=0.813, d=0.07)$, nor

244 in mean scores for the Vocabulary $(t(45)=-0.698, p=0.489, d=0.20)$ and for the Digit Span 
$245(t(45)=0.507, p=0.615, d=0.15)$ subtests. In addition, there was no difference between the

246 groups in the subscales of the YPI $(p>0.375)$.

Source-monitoring task results

248 The mixed ANCOVA conducted on the d-prime scores, with group (CP vs. non-CP) as

249 between group factor and gender as covariate, revealed a significant effect of the reading 250 condition $\left(F(1,44)=9.959, p=0.004\right.$, partial $\left.\eta^{2}=0.178\right)$, suggesting that independently of the 251 group, items read silently were less accurately recognized. In addition, the results suggested a 252 triple interaction effect between the group, the reading condition, and the type of item $(F(1$, $25344)=2.294, p=0.031$, partial $\left.\eta^{2}=0.104\right)$, suggesting that the relation between the reading 254 condition and the item type differs across the groups. To follow up the interaction effect, 255 simple effects were analyzed, revealing that the non-CP group showed a less accurate 256 recognition for the words read silently in comparison with the words read overly $(F(1,44)=$ $25716.036, p<0.001$, partial $\left.\eta^{2}=0.272\right)$. The same pattern was observed for the CP group $(F(1$, $25844)=4.981, p=0.031$, partial $\left.\eta^{2}=0.104\right)$. The CP group also showed a less accurate recognition 259 for the non-words read silently, compared to the non-words read overly $(F(1,44)=13.373$, $260 p=0.001$, partial $\left.\eta^{2}=0.237\right)$. These results are presented in the Figure 2.

261 The results of mixed ANCOVA conducted on the monitoring bias scores for each type of 262 item, with group (CP vs. non-CP group) as between factor and gender as covariate, did not 263 reveal any significant effect $(p>0.145)$.

\section{Discussion}

265 The present study investigated self-monitoring performances in a group of incarcerated 266 adolescents (IA), in comparison to a group of community adolescents (CA). We employed a 267 task assessing source monitoring of self-generated speech, which included stimuli of different 268 levels of cognitive effort (words-low effort; non-words-high effort). The task yields a 
recognition score (d prime) and two self-monitoring bias scores, internalizing bias score,

270 defined as the tendency to identify overtly read items as silently read, and externalizing bias

271 score, defined as the tendency to identify silently read items as overtly read. In light of the

272 relevant literature, the results will be discussed in relation to the psychological and clinical

273 characteristics of each group.

$274 \quad$ IA vs. CA groups

275 Firstly, no group differences were found for the d prime scores, suggesting that IA

276 participants conserve intact recognition capacities. Regarding the source monitoring bias

277 scores, the IA presented more overall biases in comparison to the CA group. Biases in the

278 source monitoring might be explained by impairments in the integration of contextual

279 information into a coherent whole and impairments in the integration of sensory information

280 previously reported in antisocial individuals (Assadi et al., 2007; Faruk et al., 2016; Hamilton,

281 Racer, \& Newman, 2015; Lindberg et al., 2004; Wang et al., 2016).

282 Interestingly, the results suggest that the two adolescent groups present different patterns of

283 monitoring bias, depending on the cognitive effort required by the material. Firstly, the IA

284 group shows a greater externalizing bias for non-words, compared to the CA group. The

285 source monitoring framework states that external attributions are more probable for stimuli

286 with increased sensorial precision (Johnson et al., 1993). We may hypothesize that, during the

287 reading phase, the IA used more sensorimotor information such as subvocalizations and failed

288 to generate the kind of cognitive information that controls generated while reading the non-

289 words. For instance, upon reading a non-word item like "TEVU", the CA group may have

290 generated idiosyncratic cognitive information (for example, one may think, "that is like T-

291 View"). In addition, accordingly to the forward model, we might explain the externalizing

292 bias for non-words as a mismatch between the predicted and the actual sensors feedback

293 (Blakemore et al., 2003). This mismatch could be due to impairments in the processing of the 
actual feedback of an action (here the silently reading of the non-words). This explanation is

295 in line with previous studies which indicate that antisocial individuals present impairments in 296 processing the sensory feedback of their actions (Hall et al., 2007; Vilà-Balló et al., 2014),

297 especially internally generated feedback (Bernat et al., 2012).

298 Contrary to non-words, word items were associated to a greater internalizing bias in the IA 299 group. We believe that the words represented items that were familiar to participants, and in 300 the IA group, familiarity may have reduced the encoding of sensory-perceptual properties of 301 the material. The source monitoring framework suggests that weak sensory-perceptual 302 precision engenders uncertainty about the "realness" of the items, which will therefore more 303 likely be attributed to an internal source (Johnson et al., 1993).

304 Contrary to the IA group, the effect of cognitive load leads to a greater tendency to internalize 305 overtly read non-words in the CA group. We may hypothesize that the CA group generated 306 more cognitive operations to encode the overtly read non-words, to the detriment of 307 sensorimotor evidence (production and sound of speech) that would have assisted in correct 308 source monitoring.

309 Regarding the relationship between psychopathic traits and source monitoring capacities, our 310 results did not reveal any association in either of the groups. This could be to the lack of 311 discriminative power of the self-report measures investigating the psychopathic dimensions, 312 which may be more thoroughly assessed through semi-structured interviews.

\section{Within group analysis of the impact of conduct problems (CP) in IA group on the source}

\section{4 monitoring abilities}

315 The results indicate that recognition scores differed between $\mathrm{CP}$ and non-CP groups, 316 depending on the item type and reading condition, the CP group showing a better recognition 317 for the non-words read aloud than for the non-words read silently. These results may suggest 318 that the CP group encodes items by favoring sensorimotor and perceptual information, which 
may yield a recognition advantage (Johnson et al., 1993). The fact that the IA with CP recognize better the non-words read aloud than those read silently might indicate that they rely more on perceptual information in the processing of the items requiring a greater amount of cognitive effort. This result may have important implications for clinical practice, in that

323 the source monitoring profile may divulge information to be considered within the assessment

324 procedure and treatment plan for IA presenting CP. However, the results did not indicate any

325 difference between the groups in monitoring bias $(p>0.168)$.

\section{Limitations}

327 Some limitations of the present study should be taken into consideration. First, the assessment 328 of psychopathic traits was performed using a self-report questionnaire, which should be 329 complemented with a semi-structured interview measure to fully assess the links between 330 psychopathy and source monitoring. Another limitation is that not all the adolescents 331 completed clinical interview, thus we could not compare the two groups regarding their 332 clinical characteristics.

\section{Conclusion}

334 To the best of our knowledge, this study is the first to investigate source monitoring in 335 delinquent adolescents. Using a self-generated speech monitoring paradigm, we observe 336 preserved recognition performances, but impairments in the source-monitoring in the IA 337 group. More precisely, the IA showed an increased externalizing bias when monitoring 338 cognitively effortful items, as well as increased internal biases when monitoring familiar 339 items. In addition, we observe that conduct problems in IA individuals may worsen their 340 recognition performances.

341 We propose that impairments in the source-monitoring abilities might contribute to limited 342 self-awareness, but also to limit insight about one's own actions and their consequences. 343 These impairments might lead to an inability to learn from their experiences and to correct 
344 their behaviors (Vilà-Balló et al., 2014). In addition, these impairments might contribute to a

345 more general tendency of the antisocial individual to experience their thoughts as real,

346 manifesting an equivalence between internal and external reality (Bateman \& Fonagy, 2016).

347 These characteristics might interfere with the motivation to change and hinder psychosocial

348 and therapeutic strategies. The present results warrant future research among IA, exploring

349 the relations between impairments in the monitoring of self-generated material and the lack of

350 insight about their behaviors, as well as the lack of responsibility for their actions.

351

352

353

354

355

356

357

358

359

360

361

362

363

364

365

366

367

368 
Achenbach, T. M. (1991). Manual for the Youth Self-Report and 1991 profile. Burlington, VT: University of Vermont Department of Psychiatry.

Achenbach, T. M., \& Rescorla, L. A. (2003). Manual for the ASEBA adult forms and profiles. Burlington, VT: University of Vermont, Research Center for Children, Youth, and Families.

Andershed, H., Kerr, M., Stattin, H., \& Levander, S. (2002). Psychopathic traits in nonreferred youths: A new assessment tool. In E. Blaauw \& L. Sheridan (Eds.), Psychopaths: Current international perspectives (pp. 131-158). The Hague, The Netherlands: Elsevier.

Assadi, S. M., Noroozian, M., Shariat, S. V., Yahyazadeh, O., Pakravannejad, M., \& Aghayan, S. (2007). Neurological Soft Signs in Mentally Disordered Offenders. Journal of Neuropsychology and Clinical Neuroscience, 19, 420-427.

Bateman, A., \& Fonagy, P. (2016). Mentalization-based treatment for personality disorders. Oxford: Oxford University Press.

Bernat, E. M., Nelson, L. D., Steele, V. R., Gehring, W. J., \& Patrick, C. J. (2011). Externalizing Psychopathology and Gain/Loss Feedback in a Simulated Gambling Task: Dissociable Components of Brain Response Revealed by Time-Frequency Analysis. Journal of Abnormal Psychology, 29, 997-1003. http://doi.org/10.1016/j.biotechadv.2011.08.021.Secreted

Blakemore, S., Oakley, D. A., \& Frith, C. D. (2003). Delusions of alien control in the normal brain. Neuropsychologia, 41, 1058-1067. http://doi.org/10.1016/S0028-3932(02)003135

Brazil, I. A., Bruijn, E. R. A. De, Bulten, B. H., Borries, A. K. L. Von, Lankveld, J. J. D. M. Van, Buitelaar, J. K., \& Verkes, R. J. (2009). Early and Late Components of Error Monitoring in Violent Offenders with Psychopathy. Biological Psychiatry, 65(2), 137143. http://doi.org/10.1016/j.biopsych.2008.08.011

Debbané, M., Van der Linden, M., Glaser, B., \& Eliez, S. (2010). Monitoring of selfgenerated speech in adolescents with 22q11.2 deletion syndrome. British Journal of Clinical Psychology, 49(3), 373-386. http://doi.org/10.1348/014466509x468223

Faruk, O., Demirel, A., Tayyib, M., \& Emül, M. (2016). Neurological soft signs in antisocial men and relation with psychopathy. Psychiatry Research, 240, 248-252. http://doi.org/10.1016/j.psychres.2016.04.094

Frick, P. J., \& White, S. F. (2008). Research review: The importance of callous-unemotional traits for developmental models of aggressive and antisocial behavior. Journal of Child Psychology and Psychiatry, 49(4), 359-375. http://doi.org/10.1111/j.14697610.2007.01862.x

Frith, C. D., Blakemore, S., \& Wolpert, D. M. (2000). Explaining the symptoms of schizophrenia : Abnormalities in the awareness of action. Brain Research Reviews, (31), 357-363.

Hall, J. R., Bernat, E. M., \& Patrick, C. J. (2007). Externalizing psychopathology and the error-related negativity. Psychological Science, 18(4), 326-333. http://doi.org/10.1111/j.1467-9280.2007.01899.x 
Hamilton, R. K. B., Racer, H. K., \& Newman, J. P. (2015). Impaired integration in psychopathy: A unified theory of psychopathic dysfunction. Psychological Review, 122(4), 770-791. http://doi.org/10.1037/a0039703

Hill, J. (2002). Biological, psychological and social processes in the conduct disorders. Journal of Child Psychology and Psychiatry and Allied Disciplines, 43(1), 133-164. http://doi.org/10.1111/1469-7610.00007

Johnson, M. K., Hashtroudi, S., \& Lindsay, D. S. (1993). Source monitoring. Psychological Bulletin, 114(1), 3-28. http://doi.org/10.1037/0033-2909.114.1.3

Jones, S. R., \& Fernyhough, C. (2006). Thought as action : Inner speech, self-monitoring, and auditory verbal hallucinations. http://doi.org/10.1016/j.concog.2005.12.003

Kaufman, J., Birmaher, B., Brent, D., Rao, U., Flynn, C., Moreci, P., ... Ryan, N. (1997). Schedule for Affective Disorders and Schizophrenia for School-Age Children-Present and Lifetime Version (K-SADS-PL): initial reliability and validity data. Journal of the American Academy of Child and Adolescent Psychiatry, 36(7), 980-988. http://doi.org/10.1097/00004583-199707000-00021

Köhler, D., Heinzen, H., Hinrichs, G., \& Huchzermeier, C. (2009). The prevalence of mental disorders in a German sample of male incarcerated juvenile offenders. International Journal of Offender Therapy and Comparative Criminology, 53(2), 211-227.

Larøi, F., Van Der Linden, M., \& Marczewski, P. (2004). The effects of emotional salience, cognitive effort and meta-cognitive beliefs on a reality monitoring task in hallucinationprone subjects. British Journal of Clinical Psychology, 43, 221-233. http://doi.org/10.1348/0144665031752970

Lindberg, N., Tani, P., Stenberg, J., \& Appelberg, B. (2004). Neurological soft signs in homicidal men with antisocial personality disorder. European Psychiatry, 19, 433-437. http://doi.org/10.1016/j.eurpsy.2004.05.011

Miall, R. C., \& Wolpert, D. M. (1996). Forward models for physiological motor control. Neural Network, 9, 1265-1279.

Moffitt, T. E. (1993). Adolesence-limited and life-course-persistent antisocial behavior:a developmnetal taxonomy. Psychological Review, 100, 674-701. Retrieved from http://graphics.tx.ovid.com/ovftpdfs/FPDDNCDCJGMHPK00/fs047/ovft/live/gv024/000 06832/00006832-199310000-00006.pdf

Moffitt, T. E., Caspi, A., Harrington, H., \& Milne, B. J. (2002). Males on the life-coursepersistent and adolescence-limited antisocial pathways: follow-up at age 26 years. Development and Psychopathology, 14(1), 179-207. http://doi.org/10.1017/S0954579402001104

Morgado, A. M., \& Vale-Dias, M. D. L. (2013). The antisocial phenomenon in adolescence: What is literature telling us? Aggression and Violent Behavior, 18(4), 436-443. http://doi.org/10.1016/j.avb.2013.05.004

Schwenck, C., Gensthaler, A., Romanos, M., Freitag, C. M., Schneider, W., \& Taurines, R. (2014). Emotion recognition in girls with conduct problems. European Child \& Adolescent Psychiatry, 23(1), 13-22. http://doi.org/10.1007/s00787-013-0416-8

Sevecke, K., Kosson, D., \& Krischer, M. (2009). The Relationship Between Attention Deficit Hyperactivity Disorder, Conduct Disorder, and Psychopythy in Adolescent Male and Female Detainees. Behavioral Sciences \& the Law, 28(2), 577-598. http://doi.org/10.1002/bsl 
Stanislaw, H., \& Todorov, N. (1999). Calculation of signal detection theory measures. Behavior Research Methods, Instruments, \& Computers, 31(1), 137-149. http://doi.org/10.3758/BF03207704

Sugimori, E., \& Tanno, Y. (2010). The effects of cognitive activity and perceptual details on speech source monitoring. British Journal of Psychology, 101, 777-90. http://doi.org/10.1348/000712610X485727

Vilà-Balló, A., Hdez-Lafuente, P., Rostan, C., Cunillera, T., \& Rodriguez-Fornells, A. (2014). Neurophysiological correlates of error monitoring and inhibitory processing in juvenile violent offenders. Biological Psychology, 102(1), 141-152. http://doi.org/10.1016/j.biopsycho.2014.07.021

Wang, X., Cai, L., Li, L., Yang, Y., Yao, S., \& Zhu, X. (2016). Neurological soft signs in Chinese adolescents with antisocial personality traits. Psychiatry Research, 243, 143146. http://doi.org/10.1016/j.psychres.2016.04.023

Wechsler, D. (1997). Manual of the Wechsler Adult Intelligence Scale-third edition (WAISIII). New York, NY: Psychological Corporation.

Wechsler, D. (2003). WISC-IV technical and interpretive manual. San Antonio, TX: Psychological Corporation. 
K-SADS-PL diagnostics

Substance abuse

Conduct disorder

Conduct disorder and substance abuse

Anxiety disorder

Conduct disorder, substance abuse and other diagnosis

(MDD, ADHD)

No diagnosis
$\%$ of the group

$10.6 \%$

$14.8 \%$

$34 \%$

$8.5 \%$

$10.6 \%$

$21.2 \%$
488

489

490

491

492

493

K-SADS-PL- Kiddie-SADS Present and Lifetime Version; ADHD- Attentiondeficit/hyperactivity disorder, MDD- Major depressive disorder

Table 2. Sample characteristics result

WISC Wechsler Intelligence Scale for Children, WAIS Wechsler Adult Intelligence Scale,

\begin{tabular}{lllll}
\hline & \multicolumn{3}{l}{ Community adolescents } & \multicolumn{2}{l}{$\begin{array}{l}\text { Incarcerated } \\
\text { adolescents }\end{array}$} \\
\hline & Mean & $S D$ & Mean & $S D$ \\
\cline { 2 - 5 } Age (years) & 15.78 & 1.60 & 15.85 & 1.30 \\
WISC/WAIS (Vocabulary) & $10.90^{* *}$ & 3.53 & $7.78^{* *}$ & 3.06 \\
WISC/ WAIS (Digit Span) & $9.04^{*}$ & 2.76 & $8.09^{*}$ & 2.44 \\
YSR (externalizing) & $56.70^{* *}$ & 9.57 & $68.54 * *$ & 7.87 \\
YSR (internalizing) & 54.12 & 10.22 & 52.60 & 10.06 \\
YPI (impulsive-irresponsible subscale) & $23.83^{* *}$ & 5.38 & $29.25^{* *}$ & 5.19 \\
YPI (CU subscale) & $29.48^{*}$ & 5.56 & $32.07^{*}$ & 7.93 \\
YPI (interpersonal problems subscale) & 28.12 & 8.74 & 31.09 & 10.27 \\
\hline YSR Youth Seff Report; YPI Yout psych & & 28.12 & &
\end{tabular}

494

YSR Youth Self Report; YPI Youth psychopathic Inventory

$495 * * \mathrm{p}<0.01$

$496 * \mathrm{p}<0.05$

497

498 Table 3. Means and standard deviations for the d prime scores in the recognition phase, of 499 both groups of adolescents

\begin{tabular}{llllll}
\hline \multirow{2}{*}{ Reading condition } & Item Type & \multicolumn{2}{c}{ Community adolescents } & \multicolumn{2}{c}{ Incarcerated adolescents } \\
\cline { 3 - 6 } & Mean & $S D$ & Mean & SD \\
\hline Overtly & Words & 2.06 & 0.65 & 1.78 & 0.70 \\
& Non-Words & 1.77 & 0.71 & 1.55 & 0.63 \\
\multirow{2}{*}{ Silently } & Words & 1.27 & 0.63 & 1.22 & 0.53 \\
& Non-Words & 1.42 & 0.74 & 1.16 & 0.65 \\
\hline
\end{tabular}

